livraisons

d'Histoire

de l'Architecture

\section{Livraisons de l'histoire de l'architecture}

$23 \mid 2012$

Varia I

\title{
Le grand chenil de Versailles sous l'Empire
}

The Great House of Hounds of Versailles during the French First Empire

Der große Zwinger in Versailles während der Empirezeit

\section{Charles-Éloi Vial}

\section{OpenEdition}

Journals

Édition électronique

URL : http://journals.openedition.org/lha/119

DOI : $10.4000 /$ lha. 119

ISSN : 1960-5994

Éditeur

Association Livraisons d'histoire de l'architecture - LHA

Édition imprimée

Date de publication : 15 juin 2012

Pagination : $35-46$

ISSN : $1627-4970$

Référence électronique

Charles-Éloi Vial, «Le grand chenil de Versailles sous l'Empire », Livraisons de l'histoire de l'architecture [En ligne], 23 | 2012, mis en ligne le 15 juin 2014, consulté le 20 avril 2019. URL : http:// journals.openedition.org//ha/119; DOI : 10.4000/lha.119

Ce document a été généré automatiquement le 20 avril 2019

Tous droits réservés à l'Association LHA 


\title{
Le grand chenil de Versailles sous l'Empire
}

\author{
The Great House of Hounds of Versailles during the French First Empire \\ Der große Zwinger in Versailles während der Empirezeit
}

\section{Charles-Éloi Vial}

1 Les chasses de Napoléon Bonaparte sont peu connues, même si dès l'an XI, il se dota d'un équipage de chasse. Cette appropriation d'un symbole d'autorité monarchique était un moyen de distraire proches, invités de marque, et aussi de prendre de l'exercice. Mais si le recrutement de veneurs issus des anciens équipages du prince de Conti et de Louis XVI ne posa pas de difficultés, il n'en fut pas de même de la logistique qu'impliquaient les chasses consulaires. Dès 1802, Bonaparte fit acheter pour son épouse le pavillon de chasse du Butard, construit par Jacques-Ange Gabriel, et le domaine du Clos-Toutain, tous deux situés en forêt de Saint-Cloud, près de la Malmaison, qui servirent à la fois de rendez-vous de chasse et de logement des veneurs. Il s'agissait, dès le départ, d'une solution de fortune, une réutilisation de bâtiments anciens. En 1804 fut créé l'office de grand veneur, confié au maréchal Alexandre Berthier. La chasse fut au centre des festivités avant et après le sacre, à Fontainebleau, avec des écuries réaménagées à la hâte, et à Paris.

2 Ces chasses diffèrent pourtant de celles, plus raffinées, de Louis XVI, Napoléon ne souhaitant pas disposer d'équipages spécialisés. Il manque la passion, car l'Empereur chasse peu et contribue à faire de la chasse une activité de cour à l'étiquette rigide. Malgré tout, des ambassadeurs et souverains furent conviés à ces chasses soigneusement organisées, dont l'écho se perpétua dans toute l'Europe. Des résidences à vocation cynégétique furent aménagées, Fontainebleau, puis Rambouillet, ainsi que des rendezvous comme La Muette, en forêt de Saint-Germain, ou le Grand Trianon. Napoléon chassa également en signe d'affirmation de son pouvoir, en Italie, en Saxe et Bavière, et même à Vienne. La pratique de la chasse à la cour du Premier empire s'imposa peu à peu dans les esprits et les habitudes. 
3 Mais la vénerie posa vite des problèmes pratiques car, si les forêts étaient suffisamment étendues, les ventes des biens nationaux avaient particulièrement touché les dépendances des résidences royales. Le logement des hommes et animaux se révela vite problématique pour le maréchal Berthier, le Clos-Toutain ne pouvant loger décemment les veneurs, rejoints par un équipage du tir, d'où une première scission de l'équipage, partiellement relogé dans les dépendances du château de Meudon, puis par la location d'écuries à La Celle Saint-Cloud, solution onéreuse et embarrassante, Napoléon convoquant souvent sur un coup de tête ses veneurs, qui devaient parcourir de grandes distances avec chiens et chevaux. Germa alors l'idée de centraliser les chasses en un seul bâtiment intégré au réseau des résidences impériales.

\section{Une solution évidente : le chenil de Versailles ? (1807-1810)}

4 Napoléon souhaitait que le budget de ses chasses ne dépassât pas les 250000 francs par an mais ce budget était grevé par des frais de location assez lourds. Le problème du logement n'affleurait pourtant que ponctuellement car, lors des déplacements de l'empereur à Rambouillet ou à Fontainebleau, une partie de l'équipage des chasses le suivait, ce qui désengorgeait le bâtiment principal, tout comme en 1808, quand Charles IV d'Espagne, exilé à Fontainebleau puis à Compiègne, se fit prêter une partie des équipages. Napoléon se préoccupa cependant peu de ce problème, étant en campagne de septembre 1806 à juillet 1807, chassant même en Pologne, sans se soucier de son porte-arquebuse égaré entre Varsovie et Thorn en transportant ses armes ${ }^{1}$. Cet éloignement des besoins de la cour, ainsi que les problèmes plus urgents de la guerre, expliquent que les soucis de son équipage ne lui parvenaient guère. Le maréchal Berthier et le baron Martial Daru, intendant de la Couronne, se préoccupèrent plus de ces questions, comme le montre leur correspondance. Au courant d'une des grandes idées de l'empereur, la réhabilitation du palais de Versailles, ils pensèrent inclure dans ce projet le déménagement de la vénerie dans les anciens locaux versaillais du Grand Chenil.

\section{Le Grand Chenil}

5 Le Grand Chenil de Versailles attira l'attention de l'administration à partir de 1807. C'était un célèbre exemple d'architecture vénatoire, parfaitement adapté aux chasses royales. L'hôtel du Grand Veneur, les logements des officiers, les écuries, chenils et bâtiments techniques cohabitaient à l'origine dans un seul ensemble architectural progressivement organisé par Louis XIV autour de l'hôtel particulier du duc de Chaulnes construit par Jules Hardouin-Mansart en 1670 derrière l'emplacement des Grandes Écuries, dans l'espace défini par l'avenue de Paris, la rue du Chenil et la rue de l'Aventure (disparues, voir ill. 2).

6 Après la construction des écuries, la tentation de disposer le long de la même avenue des institutions si complémentaires était forte, d'où le rachat par Louis XIV en 1682 de l'hôtel et de ses dépendances, réaménagés par Hardouin-Mansart. Le Grand Chenil tel qu'il se présentait alors ${ }^{2}$ était composé de l'hôtel du Grand Veneur et de deux corps de bâtiments (ill. 1), fermés par des barrières qui faisaient de l'ensemble un système articulé de dix cours de taille variable, ménageant des espaces pour les hommes et les animaux. 


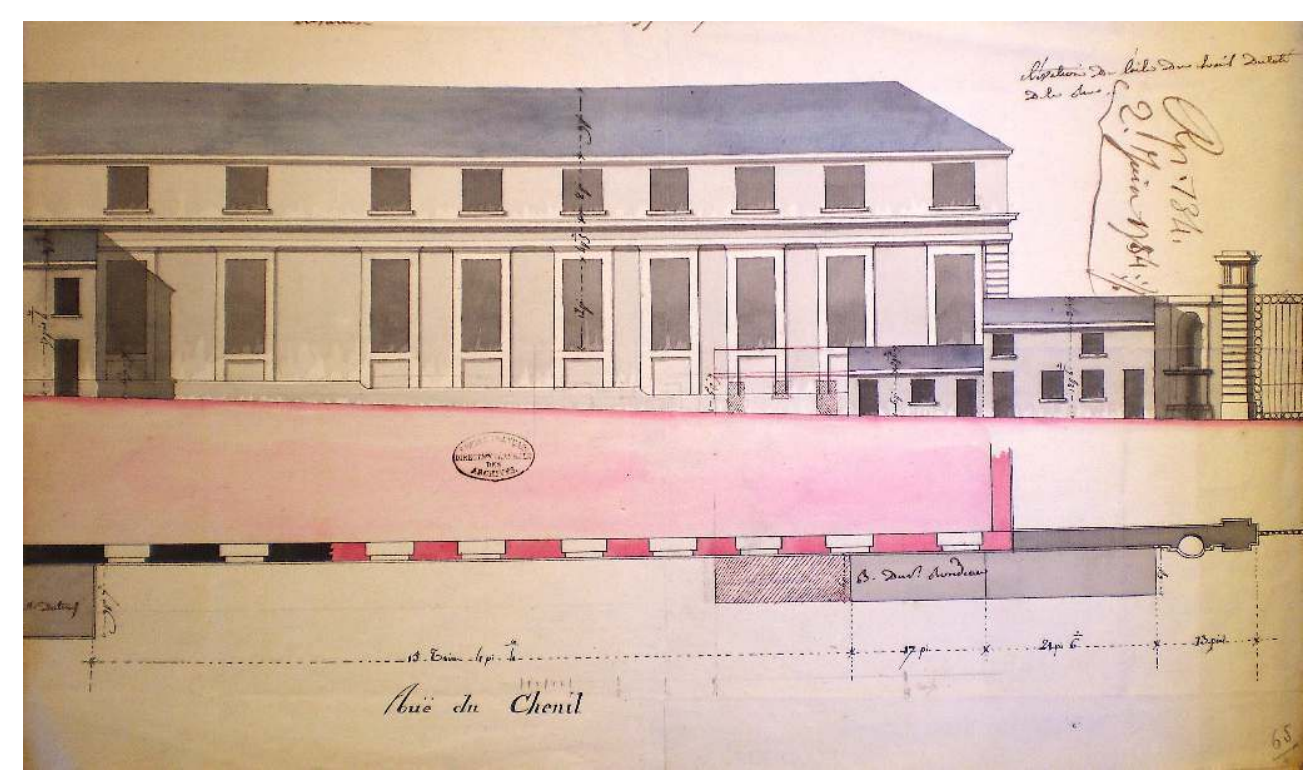

Anonyme, datée du 2 juin 1784, 36,2 × 21,3 cm., encre, lavis rose et gris. Document conservé aux Archives nationales, $0^{1} 1985^{1}$, pièce 65 .

Cl. de l'auteur.

7 L'hôtel du Grand Veneur fermait l'ensemble du côté du château et se prolongeait par une cour octogonale, un jardin et une enceinte en demi-lune en contrebas. Cet ensemble complet et cohérent fut en service jusqu'à la Révolution, bien qu'ayant un peu perdu de sa prépondérance et de son unité avec la création d'annexes sous Louis XV. En 1789, il fut entièrement vidé et en 1791 l'administration départementale s'y installa. En 1792, la chute de la monarchie rendit le sort de ces bâtiments incertains. Entre réutilisation partielle et vente au titre des biens nationaux, l'ensemble fut progressivement disloqué jusqu'au consulat. En 1806, l'hôtel du Grand Veneur était occupé par le tribunal civil de Versailles, une des deux ailes étant louée par la régie des Domaines, tandis que la seconde, tout comme les maisons de gardes, avaient été vendues à des particuliers (ill. 2). 
III. 2 : Plan du Grand chenil de Versailles d'après Gondouin, [1808], 41 x 37 cm., plume, encre noire et rouge

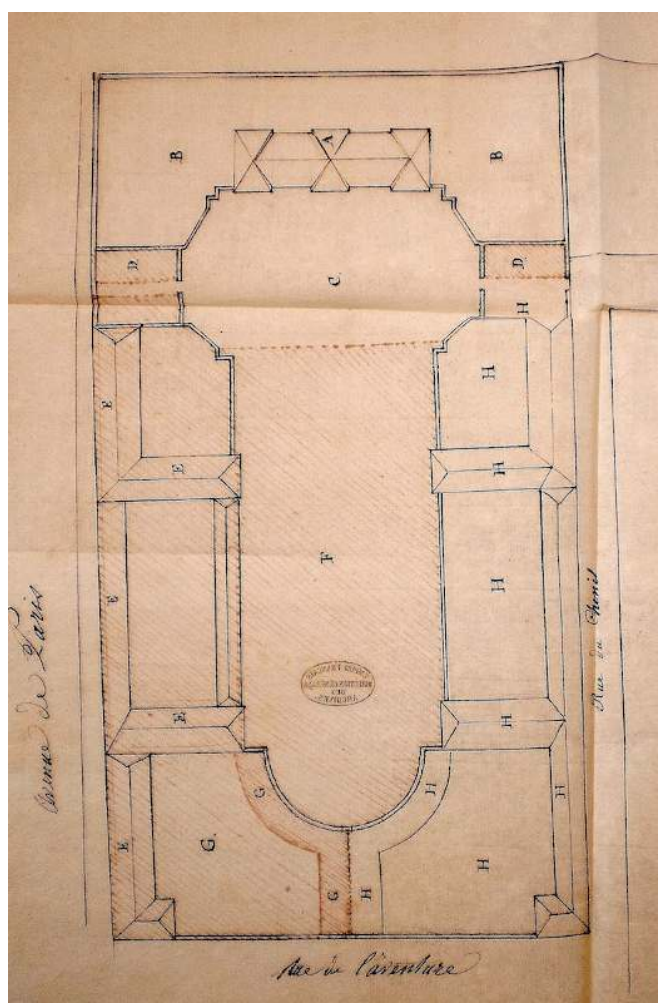

Les parties aliénées sont rayées en rouge. A : Hôtel du Grand veneur ; B : Jardin ; C : Cour d'honneur ; $D$ : Logement des portiers ; $E$ : Ancien pavillon des commandants de la vénerie ; $F$ : Cour dite « des ébats des chiens »; G : Logement des veneurs; H : Bâtiments occupés par la vénerie en 1807. Document conservé aux Archives nationales, $\mathrm{O}^{2} 328$, dossier « Versailles, vénerie et chenil ».

$\mathrm{Cl}$. de l'auteur

En 1808, cet ensemble de bâtiments aliénés fit l'objet d'un rapport synthétique destiné à en préciser la situation :

L'aile droite du chenil comprend les bâtiments où étaient logés autrefois le commandant, les officiers et les écuyers de la vénerie, et où se trouvent de grandes écuries et des remises. Ces bâtiments ont été vendus comme biens nationaux le 4 brumaire an 6 pour le prix de $654000 \mathrm{~F}$ [...]. La maison du portier qui donne sur l'avenue de Paris a été vendue par le département le 27 fructidor an 8 pour une somme de $30000 \mathrm{~F}$ [...]. La maison de portier qui donne dans la rue St. Pierre a été vendue le 14 vendémiaire an 5 au S. David pour le prix de $4500 \mathrm{~F}^{3}$.

\section{Une réinstallation partielle (1807-1809)}

9 Quelques mois même avant le rapport de Faget de Baure, rapporteur du contentieux de la Couronne, l'administrateur des Domaines, Jean-François-Thomas Goulard, expédia le 13 mai 1807 au général Duroc, grand maréchal du palais, un rapport sur les bâtiments, départ d'une réinstallation partielle dans les parties non aliénées ${ }^{4}$. Goulard y annonçait avoir chassé les locataires des bâtiments, mis à la disposition de Jean-Ferdinand-Élie Randon d'Hanneucourt, capitaine général des chasses, qui y entreposa sur le champ du matériel. Le 4 juin 1807, l'architecte Guillaume Trepsat dressa un devis pour des premiers travaux dans la cour de la Chapelle, s'élevant à 1610,11 francs. Une lettre de 
d'Hanneucourt exprime bien l'urgence de trouver un emplacement pour loger la vénerie, dont une partie était à Compiègne et l'autre à Saint-Cloud :

Les toiles sont comme votre Altesse le scai, dans les batiments de la Vénerie à Versailles, il est juste que le piqueur de cette établissement soit au prest pour les soigner. La lingerie ne peut tenir non plus au Clos-Toutain, les draps même sont dans ce moment-cy sur des chaises dans ma chambre faute d'emplacement; à Versailles la moitié de la vénerie n'est point vendu, il faudrait de toutte necessité que votre Altesse demande que cela rentre à son service, pour y placer tels que les deux établissements les choses qui n'ont pas besoin d'être sous la main. J'ai demandé le devis cy-joint à $\mathrm{M}$. Trepsat pour que Votre Altesse puisse l'examiner et que M. Daru l'aïant dans les mains donne de suitte les ordres nécessaires. Faute de ce surcroit de logement il faudrait encore louer en plus dans les villages voisins, ce qui est ruineux ${ }^{5}$.

En pleine entrevue de Tilsitt, le maréchal Berthier transmit la demande de d'Hanneucourt au comte Daru qui fit presser les travaux ${ }^{6}$ qui allaient seulement permettre l'accueil d'un piqueur, de la blanchisseuse de la vénerie, ainsi que des toiles servant à chasser le sanglier. Le fonds pour l'entretien du château de Versailles fut sollicité afin de payer ces travaux, ce qui montre bien l'idée que le château et ses dépendances formaient un ensemble cohérent, qui devait être restauré en bloc en vue d'une installation de la Cour. On retrouve la même idée avec le devis général dressé par l'architecte Jacques Gondouin pour la restauration globale du château, remis en 1807 à Napoléon ${ }^{7}$. Dans ce devis, la réhabilitation de la partie non aliénée du Chenil était estimée à 158050 francs ${ }^{8}$.

L'installation de la vénerie à Versailles pâtit donc jusqu'en 1809 des hésitations de Napoléon au sujet du sort du château. Il est important de noter que le comte Daru proposait de faire du chenil un chantier-test préalable à la réhabilitation de tout l'ensemble palatial : « Mais en attendant [que le ministre des Finances] ait soumis à Votre Majesté un plan général pour le rachat des domaines aliénés de la Liste civile, il semble convenable d'en faire un premier essai dans le rachat des parties aliénées du chenil de Versailles. »Ce rapport daté de juin 1808, ainsi qu'un projet de décret attribuant le chenil au grand veneur, proposait d'y installer la vénerie après le séjour d'automne à Fontainebleau', mais Napoléon ne signa pas, ajournant sa décision. Les chances de rachat paraissaient bloquées, le lieutenant des chasses, le baron de Cacqueray, réclama en vain un logement, qui ne lui fut accordé sous la pression du maréchal Berthier qu'au début de l'année 1809, pour une somme de 2400 francs $^{10}$.

Daru précisa par ailleurs au même moment à Berthier que « S.M. en signant le 28 octobre le budget pour les dépenses de sa maison en 1809 a cru devoir ajourner jusqu'à son retour l'exécution du projet général pour le rétablissement de cette résidence $»^{11}$. Napoléon témoigna pour le chenil de Versailles d'une indécision surprenante, dont il semble avoir souvent fait preuve en matière de bâtiments ${ }^{12}$, ajournant, reculant devant les dépenses et les projets trop ambitieux, peut-être car trop souvent absent et accaparé par d'autres problèmes avant 1809.

\section{Une restauration rapidement menée (1810-1813)}

\section{Un contexte plus favorable}

13 Après son divorce en 1809, Napoléon se retrouva confronté au problème de la vénerie. Il tint à conserver les bâtiments où logeaient ses veneurs ${ }^{13}$ mais ceux-ci étaient désormais 
enclavés dans les bois de Malmaison, appartenant à Joséphine. Pour ne pas avoir à chasser sur les terres de son ex-épouse, Napoléon devait donc trouver une nouvelle résidence de loisir assortie d'un espace cynégétique. Son choix se porta rapidement sur Trianon, où il se retira après son divorce et où le goût de la chasse lui vint très vite : il y chassa à courre comme à tir, prit plusieurs décrets relatifs à la vénerie, prépara la campagne d'acquisitions de terrains de l'année 1810, et la réforme du statut des biens de la Couronne voté à la fin du mois de janvier. L'attention de Napoléon se porta sur le Grand chenil de Versailles au moment où son goût pour la chasse, son désir de possession foncière et son intérêt pour le château étaient à leur maximum.

En 1810, Napoléon racheta les bâtiments aliénés du Chenil. Ce rachat bénéficia de la création de l'Intendance des bâtiments ${ }^{14}$, où fut nommé Costaz, qui eut sous ses ordres les architectes des palais impériaux et fut chargé de diriger les chantiers, assisté d'un comité consultatif, chargé d'inspecter les travaux et d'amender les demandes de paiement des entrepreneurs. La volonté impériale se fait sentir dans la rapidité des événements. Le 7 mars, Daru présenta un nouveau rapport favorable au rachat de la Vénerie, estimée par Goulard à 130388 francs, que Napoléon approuva ${ }^{15}$. Le contrat fut signé peu après le 26 mai 1810, après être passé entre les mains du comité du Contentieux de la Maison de l'empereur le 28 avril, le prix ayant fait l'objet de négociations assez âpres et l'expulsion des locataires ayant posé quelques problèmes ${ }^{16}$. Une des deux maisons de gardes fut rachetée en septembre 1810 pour 25500 francs $^{17}$.

\section{Le gros œuvre}

Napoléon géra aussi la restauration, en éclatant les responsabilités. Trepsat ne garda que Trianon, Alexandre Dufour fut nommé architecte du château de Versailles et Auguste Famin, architecte de Rambouillet, fut chargé du Grand Commun et du Chenil ${ }^{18}$. Il avait en effet dirigé avec succès la restauration du château de Rambouillet depuis 1807, et notamment l'installation de la vénerie dans les écuries construites sous Louis XVI, en en faisant une demeure appréciée par Napoléon qui y chassait le cerf et le canard sauvage. Le déménagement était urgent, car les bâtiments enclavés du Butard et du Clos-Toutain menaçaient ruine, le concierge se plaignait des portes qui s'écroulaient, des papiers peints qui se décollaient dans les appartement impériaux du pavillon, et du gibier qui s'échappait par les brèches de l'enceinte ${ }^{19}$. Après la remise des plans par Trepsat, Auguste Famin établit l'estimation de l'ensemble des travaux à 431 133,19 francs ${ }^{20}$.

16 La première entreprise de Famin fut d'ouvrir un accès à la rue et de détruire quatre chenils en ruine situés dans la cour des baraques, en mai $1810^{21}$. Une première vague de travaux eut lieu dès le rachat à l'été 1810. Famin expédia une demande d'acompte de 30000 francs le 12 juillet 1810, suivie d'une autre de 21000 francs le 8 septembre, principalement pour les charpentes, la toiture et la réfection des pavements ${ }^{22}$. Dès août, il fut projeté de transférer une partie des écuries dans les bâtiments existants, qui furent toutefois jugés en trop mauvais état ${ }^{23}$. Le 12 décembre 1810, lors d'une première inspection, le comité consultatif fit un rapport positif à Costaz indiquant avoir «trouvé l'écurie de gauche dans la première cour de ces bâtiments rétablie, et les logements au dessus en état d'être occupés. Le chenil du bâtiment intermédiaire entre la $2^{\mathrm{e}}$ et la $3^{\mathrm{e}}$ cour est très avancé ainsi que les logements au dessus. Ceux à gauche dans la troisième cour sont rétablis : ces travaux nous ont parus bien exécutés ${ }^{24}$. Au $1^{\mathrm{er}}$ janvier 1811 , l'équipage du tir et du vol purent déménager dans l'aile gauche des bâtiments, avec chevaux et 
faucons, le comte Daru informant Alexandre Desmazis, administrateur du Garde-Meuble «que les équipages à tir et du vol des chasses de Sa Majesté doivent se transporter à Versailles pour s'établir dans le pavillon gauche de la vénerie $»^{25}$. Le secrétariat de la vénerie déménagea à Paris, il ne resta au Clos-Toutain que l'équipage du courre, vite partagé entre Versailles et les écuries du parc Monceau, plus près de l'empereur qui chassait de plus en plus souvent.

\section{Le tournant de 1811}

L'année 1811 vit le passage des travaux de gros œuvre aux travaux d'aménagement. Famin expédia encore le $1^{\mathrm{er}}$ mars une série de devis et de soumissions d'entrepreneurs, approuvés en bloc le 30 juillet 1811, pour 40350,14 francs, dont 20 031,43 pour la maçonnerie, 17 123,20 pour la charpente et 3195,51 pour la couverture, suivi d'un devis s'élevant à 18 869,14 francs pour la restauration des maisons de portiers, de 8881,98 francs pour l'aménagement de la sellerie ${ }^{26}$, puis 3761,46 francs pour la fauconnerie ${ }^{27}$. Tous ces travaux furent imputés sur un budget commun de 700000 francs pour les travaux de la Grande Écurie et la Vénerie, même si les deux chantiers étaient bien distincts. Dès le début de l'année, les projets d'aménagement des logements et de la salle d'armes sont élaborés. Cacqueray, déjà logé depuis 1809, vit son logement amélioré à hauteur de 2700 francs, tandis qu'Antoine de Beauterne, porte-arquebuse, bénéficia d'un logement refait à neuf.

III. 3 : Plan de la cour de l'hôtel du Grand veneur de Versailles par Famin, janvier 1811, 32 x 21,3 $\mathrm{cm}$., plume et lavis

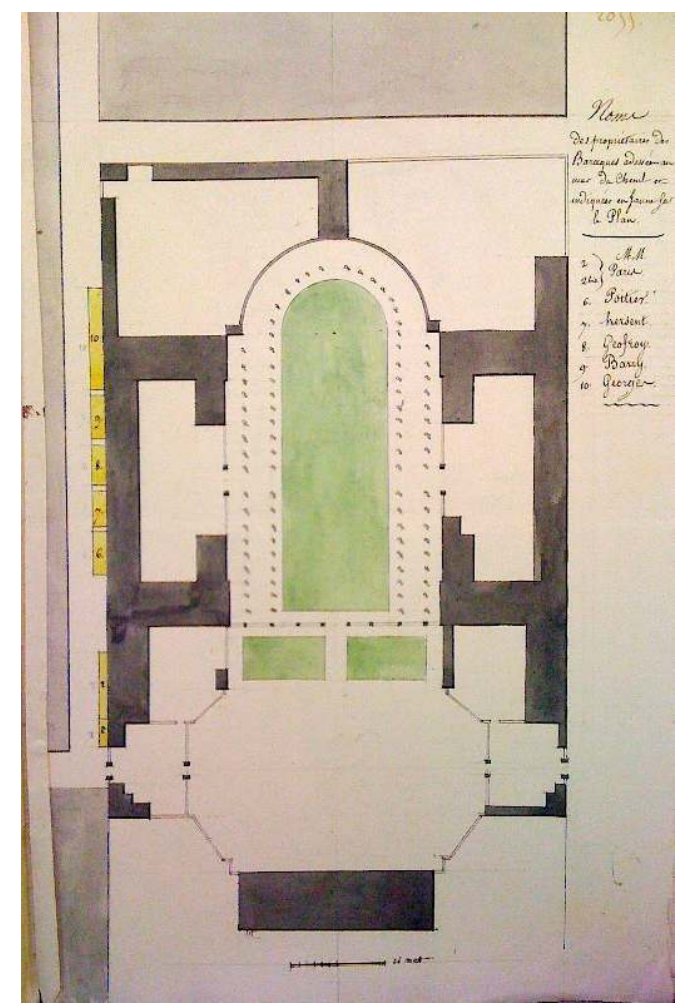

En jaune les baraques à détruire accolées au bâtiment des écuries et du fourrage. Document conservé aux Archives nationales, $\mathrm{O}^{2} 320$, dossier 6 « vénerie et chenil », joint à une lettre du 25 janvier 1811 de l'architecte Famin au baron Costaz, intendant des bâtiments.

CL. DE L'AUTEUR 
Napoléon en personne vint inspecter l'ensemble du chantier le 10 juillet 1811, comme le rapporte Fontaine : «S.M. est arrivée en calèche le soir, elle est allée sans descendre de voiture aux écuries, à la vénerie, et a fait le tour du château ${ }^{28}$. " S'il n'y trouva rien à redire, il envoya toutefois son grand veneur le 23 juillet suivant, qui se déclara, à l'exception d'une écurie située dans l'ancienne chapelle ${ }^{29}$, satisfait du travail, malgré des remarques sur la répartition des espaces entre les équipages ainsi que sur des logements. Le 2 août, une lettre de Famin fit écho de la demande du grand veneur de disposer d'un plan au sol des bâtiments qui devait lui permettre de décider lui-même des

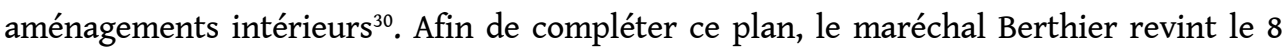
août et écouta les conseils du chevalier de Beauterne, veneur âgé et expérimenté ${ }^{31}$, qui le conseilla pour l'aménagement des écuries ou le rétablissement des bassins. Le plan fut achevé et signé par Berthier le 28 août (ill. 4 et 5), expédié le 6 septembre ${ }^{32}$.

III. 4 : Plan avec retombes du Grand chenil de Versailles par Famin, annoté et paraphé par Berthier le 28 août 1811, 89,4 x 62 cm., encre, lavis rose, et vert. Document conservé aux Archives nationales, Cartes et plans, VA, boîte XXVIII, pièce 3 .

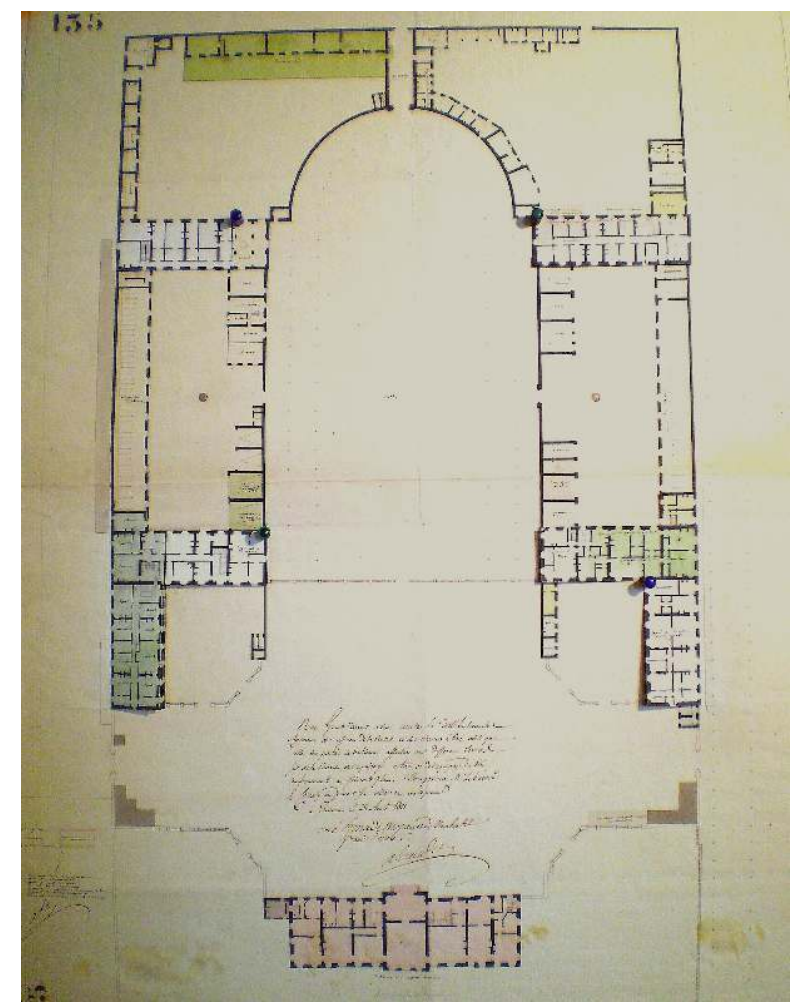

En rose, l'Hôtel du Grand veneur, en vert le service du tir et de la fauconnerie, en blanc celui du courre Cl. de l'auteur 
III. 5 : Détail d'une retombe . Document conservé aux Archives nationales, Cartes et plans, VA, boîte XXVIII, pièce 3

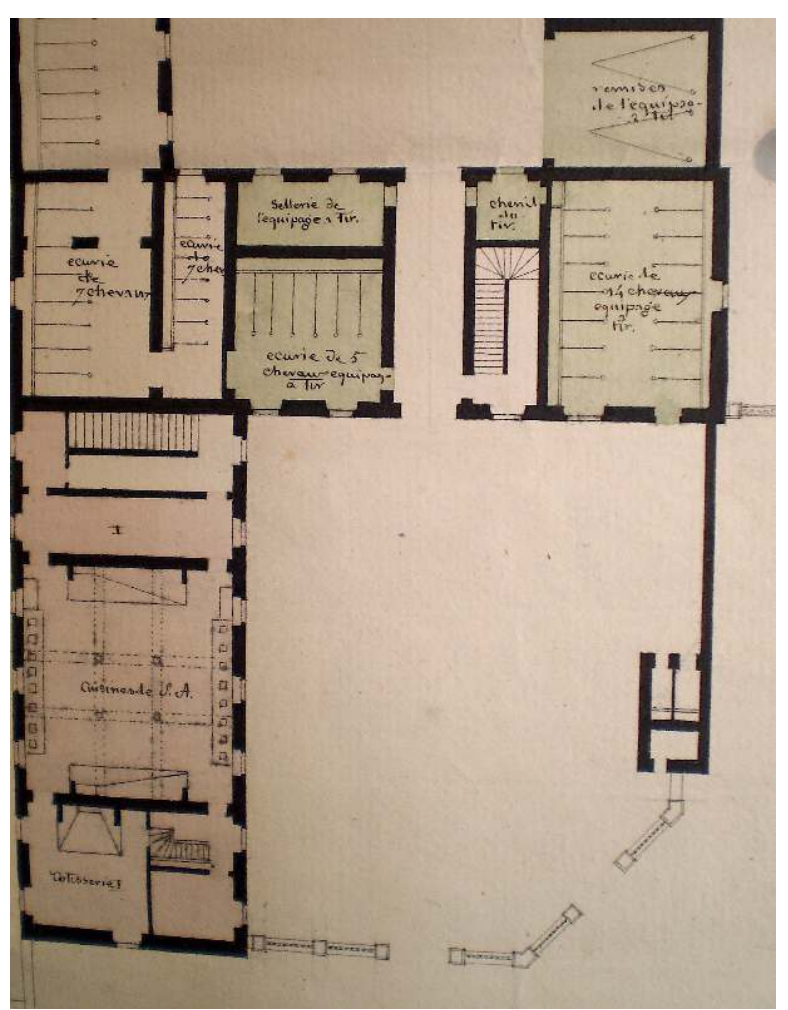

En rose, les cuisines, en vert l'écurie, la sellerie, le chenil et une remise de l'équipage du tir, en blanc les écuries de l'équipage de chasse à courre

Cl. de l'auteur.

Il servit de base de travail à Famin pour la réhabilitation des logements des officiers ${ }^{33}$. Il expédia un dernier devis pour 191 113,19 francs ${ }^{34}$, dernière tranche des travaux entamés en 1810. Les chevaux des écuries furent peut-être alors peints par Théodore Géricault dans le cadre de ses études de chevaux ${ }^{35}$.

Le Chenil fut aussi sécurisé par la démolition des baraques qui s'étaient greffées en toute illégalité sur son flanc (ill. 3), et qui présentaient des risques d'incendie du magasin des fourrages mitoyen ${ }^{36}$. Suite à des vols fin 1811, le maréchal Berthier fit presser la réoccupation des logements ${ }^{37}$.

\section{Des finitions}

Signe que les travaux s'achevaient, les budgets ne furent pas tous épuisés. En 1812, les logements des officiers furent complétés sur le budget de 1811, pour un montant de 10000 francs. Le fonds d'entretien de 200000 francs de 1812 fut en revanche sollicité pour l'infirmerie des chevaux dont le devis fut dressé le 11 juillet 1812 pour 6853 francs $^{38}$. Il ne restait en 1812 en gros œuvre que la mise en état des façades, des croisées, du fournil, ainsi que des travaux de couverture. Le comité consultatif se déclara le 4 mars 1813 satisfait de ces travaux. La dernière maison de garde fut enfin rachetée le 24 décembre 1812 pour 24000 francs $^{39}$, complétant l'ensemble. Famin demanda 59850 francs pour sa réfection, 6000 francs pour les façades ${ }^{40}$ et 190190 francs pour l'entretien 
courant ${ }^{41}$. Le 5 octobre suivant, les travaux de l'année 1813 furent qualifiés comme « de peu d'importance $»^{42}$.

Conduite avec célérité, la réinstallation de la vénerie dans le centre originel de Versailles fut un tel succès qu'à leur retour, les Bourbons trouvèrent un bâtiment abritant 150 chevaux, 300 chiens et une cinquantaine de veneurs, ainsi qu'un service des chasses fonctionnel. Cependant, il ne s'agissait pas pour autant pour Napoléon de renouer avec l'Ancien Régime. Ce n'est qu'à partir du moment où il désira chasser et résider à Trianon qu'il prit en compte les besoins de sa vénerie. Le sort du Grand Chenil est ainsi proche de celui de nombreux bâtiments impériaux, restaurés à l'occasion du Sacre, délaissés jusqu'en 1807, moment où naissent des grands projets comme le réaménagement du château de Compiègne, concrétisés à partir de 1810. Sur une autre échelle, la plupart des bâtiments dédiés aux chasses connurent le même sort, par exemple les pavillons, meublés simplement jusqu'à l'éveil de l'intérêt de Napoléon pour la chasse, d'où des aménagements somptueux, à Bagatelle et Trianon. Paradoxalement, la création d'une vénerie et la réhabilitation du chenil de Versailles sont représentatives du pragmatisme de Napoléon, plus que de son goût pour le luxe ou de sa fascination par la monarchie.

\section{NOTES}

1. Arch. nat., $\mathrm{O}^{2} 128$, fol. 78r.

2. Voir Jean-Aymar Piganiol de La Force, Nouvelle description des châteaux et parcs de Versailles et de Marly, contenant une explication historique de toutes les peintures, tableaux, statues, vases et ornements qui s'y voient, Paris, Aumont, 1764, vol. 1, p. 3.

3. Paris, Bibliothèque nationale de France, département des manuscrits, ms. fr. 6583, fol. 9r à 12r, rapport de Faget de Baure à Daru, 9 juin 1808.

4. Arch. nat., $\mathrm{O}^{2}$ 330, chemise 17, pièces 1 et 2, rapport de Goulard à Duroc, Paris, 13 mai 1807.

5. Arch. nat., $\mathrm{O}^{2} 330$, chemise 9 , pièces 1 et 2, lettre de d'Hanneucourt à Berthier, Compiègne, 16 juin 1807, et devis de Trepsat, 4 juin 1807.

6. Arch. nat., $\mathrm{O}^{2} 330$, chemise 17, pièces 6 et 7, lettre de Berthier à Daru, Tilsitt, 29 juin 1807, et lettre de Daru à Trepsat, Königsberg, 17 juillet 1807 (minute).

7. Voir Jérémie Benoît, Napoléon et Versailles, Paris, R.M.N., 2005, p. 20-21.

8. Arch. nat., $\mathrm{O}^{2} 328$, dossier «Versailles, vénerie et chenil », «Extrait du travail général de M. Gondouin sur la dépense nécessaire pour la restauration de Versailles », avec un calque du plan du chenil, plume, encre noire et rouge, 41 sur $47 \mathrm{~cm}, 13$ mai 1808 .

9. BnF, ms. fr. 6583, fol. 13r, rapport de Daru à Napoléon, Paris, 29 juin 1808 et 15r, projet de décret antidaté du 1er décembre 1808.

10. Arch. nat., $\mathrm{O}^{2} 330$, chemise 8 , «Versailles, mise en état d'appartement dans la vénerie demandée par M. d'Hanneucourt ", pièce 2, lettre de Faget de Baure à Trepsat, minute, s.d. [janvier 1809].

11. Arch. nat., $\mathrm{O}^{2} 330$, chemise 8 , «Versailles, mise en état d'appartement dans la vénerie demandée par M. d'Hanneucourt ", pièce 4, lettre de Daru à Berthier, minute, s.d. [janvier 1809].

12. Cette difficulté qu'avait Napoléon à prendre des décisions avec ses architectes est rapportée par plusieurs témoins. Voir par exemple dans Charles Percier, Pierre-François-Léonard Fontaine, 
Parallèle entre différentes résidences de souverains, Paris, Les auteurs, 1833, p. 121 : Napoléon ressortit de sa visite de Versailles en juillet 1811 "plus incertain encore après avoir tout vu ", avant d'ajourner sa décision.

13. Arch. nat., $O^{2} 155$, pièce 152 , décret, Tuileries, 16 décembre 1809 , copie.

14. Arch. nat., $\mathrm{O}^{2} 158$, pièce 454 , décret, Tuileries, 9 janvier 1810 , copie.

15. Arch. nat., $\mathrm{O}^{2} 156$, pièce 331, rapport de Daru à Napoléon, Paris, 7 mars 1810, apostillé "Approuvé. Nap.».

16. Arch. nat., $O^{2} 222$, p. 242.

17. Arch. nat., $\mathrm{O}^{2} 156$, pièce 362 , rapport de Daru à Napoléon, Paris, 4 septembre 1810, apostillé « Approuvé. Nap.».

18. Arch. nat., $O^{2} 158$, pièce 457 , décret, Tuileries, 8 février 1810 , copie.

19. Arch. nat., $\mathrm{O}^{2} 326$, dossier I6, lettres de Belleau, concierge du Butard, à Blondel, inspecteur des bâtiments à Saint- Cloud [1808-1810].

20. Arch. nat., $\mathrm{O}^{2} 320$, dossier 6 , "vénerie et chenil ", procès verbal de remise des plans à Famin, par Trepsat, et devis, Versailles, 10 mars 1810.

21. Arch. nat., $\mathrm{O}^{2} 320$, dossier 6 , « vénerie et chenil », lettre de Famin à Costaz, 14 mai 1810, Versailles.

22. Arch. nat., $\mathrm{O}^{2} 321$, dossier II2, lettres de Famin à Costaz du 12 juillet et du 8 septembre 1810.

23. Arch. nat., $\mathrm{O}^{2} 226$, dossier I, « Intendant général », lettre de Daru à Costaz, Paris, 8 août 1810.

24. Arch. nat., $\mathrm{O}^{2} 254$, dossier I, « visites annuelles », séance du 12 décembre 1810.

25. Arch. nat., $\mathrm{O}^{2} 555$, dossier 2, «Versailles 1811 », pièce 54, lettre de Daru à Desmazis, administrateur du Garde-Meuble de la Couronne, Paris, 3 janvier 1811.

26. Arch. nat., $\mathrm{O}^{2} 237$, dossier I, « juillet 1811 », lettres de Costaz à Famin, 30 juillet 1811, minutes.

27. Arch. nat., $\mathrm{O}^{2}$ 249, dossier III, lettre de Costaz à Famin, Paris, 6 août 1811.

28. Pierre-François-Léonard Fontaine, Journal, 1799-1853, Paris, École nationale supérieure des Beaux-Arts, Institut français d'architecture, Société de l'histoire de l'art français, 1987, vol. 1, p. 297.

29. Arch. nat., $\mathrm{O}^{2} 235$, dossier V, « S.E. le Grand Veneur. Mr le comte de Girardin, capitaine des chasses du roi », lettre de Berthier à Costaz, Trianon, 23 juillet 1811.

30. Arch. nat., $O^{2} 320$, dossier 6, lettre de Famin à Costaz, Rambouillet, le 2 août 1811.

31. Arch. nat., $\mathrm{O}^{2} 320$, lettre de Famin à Costaz, Versailles, 8 août 1811. Le chevalier de Beauterne avait été porte-arquebuse de Louis XVI avant d'être celui de Napoléon. Il était aussi connu pour avoir aidé à tuer la « bête du Gévaudan » en 1764.

32. Arch. nat., $\mathrm{O}^{2} 235$, dossier 1, lettre de Costaz à Famin, minute, Paris, 6 septembre 1811.

33. Arch. nat., Cartes et plans, VA, boîte XXVIII, plan 3.

34. Arch. nat., $\mathrm{O}^{2} 320$, dossier 6, lettre de Famin à Costaz, 28 novembre 1811.

35. Théodore Géricault, Chevaux vus de la croupe, Paris, Musée du Louvre, huile sur toile, inv. 4891, 38 x 46 cm., v. 1812.

36. Arch. nat., $\mathrm{O}^{2} 320$, dossier 6 , "vénerie et chenil », lettre de Famin au baron Costaz, 25 janvier 1811 ; et plan de la cour de l'hôtel du grand veneur de Versailles avec en jaune les baraques accolées au bâtiment des écuries et du fourrage, 1811, 32 x 21,3 cm., plume, lavis.

37. Arch. nat., $\mathrm{O}^{2} 233$, dossier $\mathrm{I}$, « janvier 1812 », lettre de Costaz à Berthier, 20 janvier 1812, minute.

38. Arch. nat., $\mathrm{O}^{2} 234$, dossier 1 , lettres de Costaz à Famin, 11 juillet 1812, minutes.

39. Arch. nat., $\mathrm{O}^{2} 243$, dossier III, rapport de Mounier à Champagny, 2 février 1813.

40. Arch. nat., $\mathrm{O}^{2} 327$, dossier 3, projet de budget pour 1813, Famin à Costaz, Versailles, 3 août 1812.

41. Arch. nat., $\mathrm{O}^{2} 320$, dossier 2 , pièce 15 .

42. Arch. nat., $\mathrm{O}^{2}$ 253, dossier I4, « visites annuelles, 1813 », rapport du 5 novembre 1813. 


\section{RÉSUMÉS}

Dès le Consulat, la réapparition progressive d'un phénomène de cour autour de Napoléon Bonaparte entraîna de nombreux aménagements architecturaux, ainsi que la réhabilitation et la remise en service de nombreux bâtiments autrefois construits pour le service des rois de France qui avaient survécus à la Révolution française. Si les travaux aux Tuileries, à Saint-Cloud et à Fontainebleau furent vivement menés de 1802 à 1804, d'autres chantiers prirent beaucoup plus de temps à aboutir, comme ceux de Rambouillet, Trianon et Compiègne à partir de 1805. Parmi les projets d'installation de la cour impériale qui n'aboutirent jamais, le plus ambitieux, celui de Versailles, est particulièrement représentatif du mécanisme de réinstallation mis en place par Napoléon $\mathrm{I}^{\mathrm{er}}$. La remise en service des dépendances précéda ainsi celle du château, qui ne fut jamais achevée. Le Grand Chenil de Versailles, qui avait hébergé les équipages des chasses royales depuis Louis XIV, finit ainsi par abriter à partir de 1810 les services de la vénerie impériale, suite à une campagne de travaux exemplaire et efficacement menée à partir de 1810 et jusqu'en 1813, alors même que Napoléon abandonnait progressivement l'idée de s'installer à Versailles et concentrait ses efforts sur la rénovation du Grand Trianon.

Once the French Consulate came into power, the progressive re-emergence of a court phenomenon focussed on Napoléon Bonaparte entailed numerous architectural projects, such as restoring and re-establishing the use of numerous buildings which were originally built for the monarchy, and which had survived the French Revolution. While work at the Tuileries, at SaintCloud, and at Fontainebleu was energetically carried out between 1802 and 1804, much more time was required to complete other restoration projects, such as those at Rambouillet, Trianon and Compiègne, which began in 1805. Among the never-completed projects to establish the imperial court, the most ambitious one, that of Versailles, is particularly representative of the system of restoration instituted by Napoléon I. The outbuildings were thus restored to active service before the palace itself, on which work was in fact never completed. The Great House of Hounds of Versailles, which had housed royal hunting crews since Louis XIV, thus came to accommodate the crew of the imperial hunt from 1810 onwards, thanks to an exemplary programme of renovations efficiently carried out from 1810 until 1813, at which point Napoléon was increasingly abandoning the idea of establishing himself at Versailles and concentrating his efforts on the renovation of the Grand Trianon.

$\mathrm{Ab}$ der Zeit des Konsulats entwickelte sich allmählich um Napoleon Bonaparte ein Hofphänomen, welches Anlass zur Errichtung neuer Bauten wurde wie ebenso zu Umgestaltung, Modernisierung und Wiederinbetriebsetzung von etlichen Bauten führte, die damals im Dienste der französischen Könige gestanden und die Französische Revolution überlebt hatten. Die Umbauarbeiten der Tuilerien, von Saint-Cloud und Fontainebleau geschahen zwischen 1802 und 1804 rasch, dagegen wurden sie ab 1805 in Rambouillet, Trianon und Compiègne verzögert. Unter den Bauplänen des kaiserlichen Hofes, die nie zu Stande kamen, gilt das ansehnliche Umbauprojekt von Versailles als besonders repräsentativ, den Willen Napoleons zur Wiedereinrichtung aufzuzeigen. Noch vor dem Schlossumbau kamen die Nebengebäude wieder in Betrieb. Die Umbauarbeiten am Schloss hingegen wurden nie zu Ende geführt. Der große Zwinger von Versailles, der die Jagdmannschaften der Könige seit Ludwig XIV. beherbergt hatte, sollte nun die Dienste der 
kaiserlichen Hetzjagden unterbringen, als Folge einer vorbildlichen Baukampagne, die von 1810 bis 1813 effizient geführt wurde. Gleichzeitig verzichtete aber Napoleon allmählich auf die Idee, sich in Versailles niederzulassen und konzentrierte sich auf die Renovierung des großen Trianon.

\section{AUTEUR}

\section{CHARLES-ÉLOI VIAL}

Charles-Éloi Vial, né en 1987, est conservateur stagiaire à l'École nationale des chartes. Auteur d'un mémoire de master 2 de l'Université Paris-IV Sorbonne, intitulé « Napoléon et la chasse », dir. de Jacques-Olivier Boudon, il a soutenu une thèse d'École des chartes sur la vénerie à la Cour du Premier Empire, dirigée par Christine Nougaret.

Adresse électronique : charles-eloi.vial@enc.sorbonne.fr 